\title{
The Relationship Between Magnetic Helicity and Current Helicity
}

\author{
J.H. Liu, Y. Zhang \\ and H.Q. Zhang \\ ${ }^{1}$ National Astronomical Observatories, Chinese Academy of Sciences, Beijing 100012, China \\ email: ljh@sun10.bao.ac.cn
}

\begin{abstract}
We have studied the magnetic helicity transport rate and the current helicity for solar active region (AR) NOAA 10488 and find a complex relationship between them. We further extend this study to a statistical one, and find that 33 among the selected 57 ARs show same sign for the two parameter.
\end{abstract}

Keywords. Sun: activity, magnetic fields

\section{Observation and method}

With the vector magnetograms from Huairou Solar Observing Station (HSOS) of the National Astronomical Observatories of China, longitudinal current helicity $B_{\|} \cdot\left(\nabla \times B_{\|}\right)$ had been calculated. And using the 96 minute cadence longitudinal magnetograms taken by the Michelson Doppler Imager (MDI) on board the Solar and Heliospheric Observatory $(\mathrm{SOHO})$, we calculated the magnetic helicity transporting rate $d H_{\text {corana }} / d t=$ $-2 \oint_{\text {photohpere }}\left(A_{p} \cdot V_{\perp}\right) B_{n} d^{2} x$ (Démoulin and Berger 2002) with the local Correlation Tracking method (LCT, Chae et al. 2001). We have gathered 5 days of observing data for each AR. Before applying the LCT method to the MDI magnetograms, we do the nonlinear mapping, flux density interpolating and the geometrical foreshorten correcting works according to Chae et al. (2001).

\section{Result}

We calculate the magnetic helicity transport rate and the current helicity of AR NOAA 10488 who has passed across the solar disk from Oct 26 to Nov 3 (Zhang, et al. 2003). Figure 1 shows the comparison between the spatial distribution of $-2\left(v \cdot A_{p}\right) B_{z}$ and the current helicity density. The former is a measure of the local contribution of the foot point motion to the rate of the magnetic helicity transport, and the latter is a measure of the photospheric helicity state. So the figure implies that the current helicity at the photosphere changes with the transfer of magnetic helicity from the sub-atmosphere in the emerging process of magnetic flux. Figure 2 shows the temporal variations of $d H / d t$ and average current helicity in AR 10488. The data is gathered from Oct 27 to 30 when the AR was not far from the disk center. We can see that the magnetic helicity transport rate was negative, and the average current helicity decreased weakly with time.

Figure 3 shows the statistical relationship between magnetic helicity transport rate and current helicity imbalance $\rho_{h}=\sum h_{c} / \sum\left|h_{c}\right|$ (Bao and Zhang 1998) of 57 ARs. We see a weak same-sign trend. It should be pointed that the ARs we selected were bi-pole or multi-pole ARs and showed not obviously hemispheric preference for both helicity. 


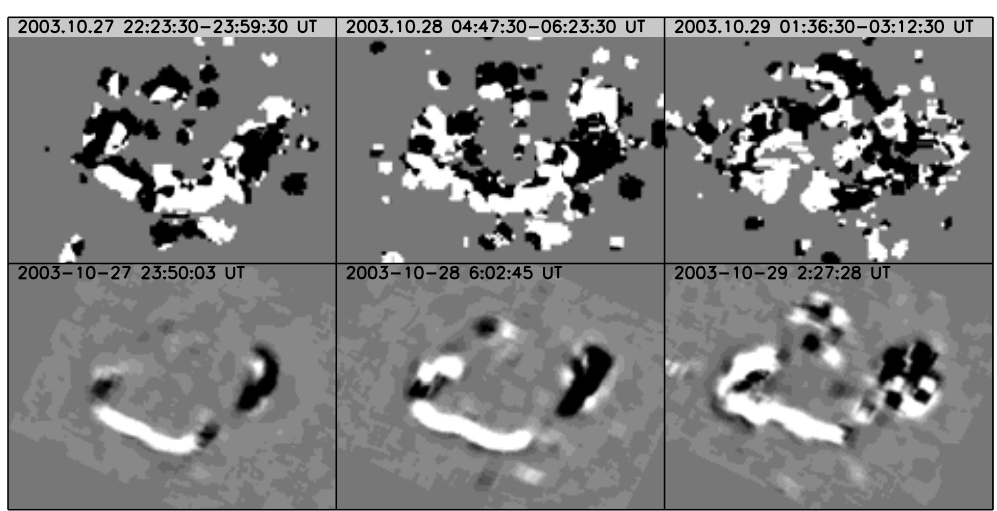

Figure 1. Gray-scale map of $-2\left(v \cdot A_{p}\right) B_{z}$ (upper) and $B_{\|} \cdot\left(\nabla \times B_{\|}\right)$(lower) of AR10488.
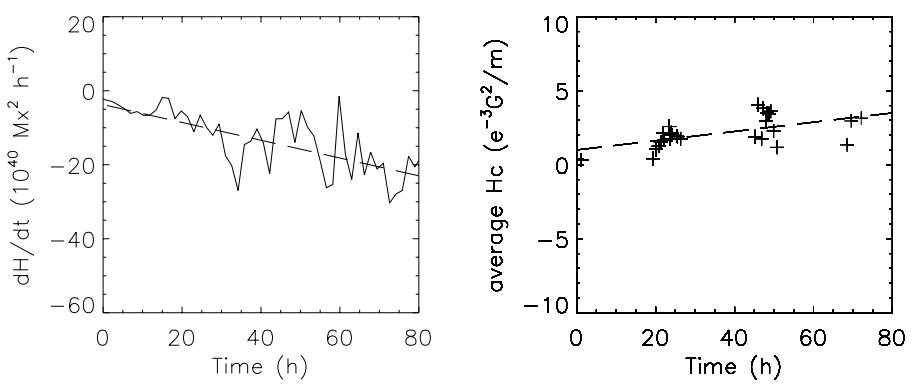

Figure 2. Measured helicity transport rate as a function of time (left) and time variation of average current helicity (right) of AR 10488

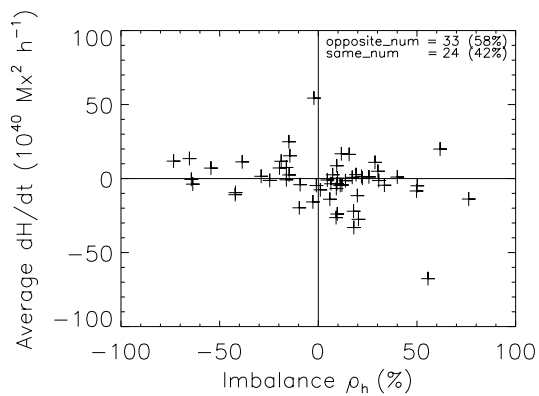

Figure 3. comparison of average $d H / d t$ and $\rho_{h}$ for 57 ARs

That the two parameters of 10488 and other ARs have same-sign means that the rotation of sunspots synchronize with the twist of vector magnetic field. It reflects the complexity of the helicity injection and the interaction of magnetic configuration.

\section{Acknowledgements}

We thank the staff in Huairou group for their discussions. We are grateful to all members of the SOHO teams for providing the wonderful data.

\section{References}

Bao, S.D. and Zhang, H.Q. 1998, ApJ 496, L43

Chae, J. 2001, ApJ 560, L95

Démoulin, P. and Berger, A. 2002, SoPh 215, 203

Zhang, H.Q., Bao, X.M., Zhang, Y., Liu, J.H., Bao, S.D., Deng, Y.Y., et al. 2003, ChJAA 3, 491 